DESY 06-036

hep-ph/0604053

DCPT/06/40, IPPP/06/20

April 2006

\title{
Next-to-Next-to-Leading Order Evolution of Non-Singlet Fragmentation Functions
}

\author{
A. Mitov ${ }^{a}$, S. $\operatorname{Moch}^{a}$ and A. Vogt ${ }^{b}$ \\ ${ }^{a}$ Deutsches Elektronensynchrotron DESY \\ Platanenallee 6, D-15735 Zeuthen, Germany \\ ${ }^{b}$ IPPP, Department of Physics, University of Durham \\ South Road, Durham DH1 3LE, United Kingdom
}

\begin{abstract}
We have investigated the next-to-next-to-leading order (NNLO) corrections to inclusive hadron production in $e^{+} e^{-}$annihilation and the related parton fragmentation distributions, the 'time-like' counterparts of the 'space-like' deep-inelastic structure functions and parton densities. We have re-derived the corresponding second-order coefficient functions in massless perturbative QCD, which so far had been calculated only by one group. Moreover we present, for the first time, the third-order splitting functions governing the NNLO evolution of flavour non-singlet fragmentation distributions. These results have been obtained by two independent methods relating time-like quantities to calculations performed in deep-inelastic scattering. We briefly illustrate the numerical size of the NNLO corrections, and make a prediction for the difference of the yet unknown timelike and space-like splitting functions at the fourth order in the strong coupling constant.
\end{abstract}


In this letter we address the evolution of the parton fragmentation distributions $D^{h}$ and the corresponding fragmentation functions $F_{a}^{h}$ in $e^{+} e^{-}$annihilation, $e^{+} e^{-} \rightarrow \gamma, Z \rightarrow h+X$ where

$$
\frac{1}{\sigma_{\text {tot }}} \frac{d^{2} \sigma}{d x d \cos \theta}=\frac{3}{8}\left(1+\cos ^{2} \theta\right) F_{T}^{h}+\frac{3}{4} \sin ^{2} \theta F_{L}^{h}+\frac{3}{4} \cos \theta F_{A}^{h} .
$$

Here $\theta$ represents the angle (in the center-of-mass frame) between the incoming electron beam and the hadron $h$ observed with four-momentum $p$, and the scaling variable reads $x=2 p q / Q^{2}$ where $q$ with $q^{2} \equiv Q^{2}>0$ is the momentum of the virtual gauge boson. The transverse $(T)$, longitudinal $(L)$ and asymmetric $(A)$ fragmentation functions in Eq. (11) have been measured especially at LEP, see Ref. [1] for a general overview. Disregarding corrections suppressed by inverse powers of $Q^{2}$, these observables are related to the universal fragmentation distributions $D^{h}$ by

$$
F_{a}^{h}\left(x, Q^{2}\right)=\sum_{\mathrm{f}=\mathrm{q}, \overline{\mathrm{q}}, \mathrm{g}} \int_{x}^{1} \frac{d z}{z} c_{a, \mathrm{f}}\left(z, \alpha_{\mathrm{s}}\left(Q^{2}\right)\right) D_{\mathrm{f}}^{h}\left(\frac{x}{z}, Q^{2}\right) .
$$

The coefficient functions $c_{a, \mathrm{f}}$ in Eq. (2) have been calculated by Rijken and van Neerven in Refs. [2-4] up to the next-to-next-to-leading order (NNLO) for Eq. (1), i.e., the second order in the strong coupling $a_{\mathrm{s}} \equiv \alpha_{\mathrm{s}}\left(Q^{2}\right) /(4 \pi)$. Below we will present the results of a re-calculation of these functions by two approaches differing from that employed in Refs. [2-4].

Besides the second-order coefficient functions, a complete NNLO description also requires the third-order contributions to the splitting functions (so far calculated only up to the second order [5-7]) governing the scale dependence (evolution) of the parton fragmentation distributions. In a notation covering both the (time-like $q, \sigma=1$ ) fragmentation distributions and the (spacelike $q, Q^{2} \equiv-q^{2}, \sigma=-1$ ) parton distributions, the flavour non-singlet evolution equations read

$$
\frac{d}{d \ln Q^{2}} f_{\sigma}^{\mathrm{ns}}\left(x, Q^{2}\right)=\int_{x}^{1} \frac{d z}{z} P_{\sigma}^{\mathrm{ns}}\left(z, \alpha_{\mathrm{s}}\left(Q^{2}\right)\right) f_{\sigma}^{\mathrm{ns}}\left(\frac{x}{z}, Q^{2}\right)
$$

with

$$
P_{\sigma}^{\mathrm{ns}}\left(x, \alpha_{\mathrm{s}}\left(Q^{2}\right)\right)=a_{\mathrm{s}} P^{(0) \mathrm{ns}}(x)+a_{\mathrm{s}}^{2} P_{\sigma}^{(1) \mathrm{ns}}(x)+a_{\mathrm{s}}^{3} P_{\sigma}^{(2) \mathrm{ns}}(x)+\ldots .
$$

The superscript 'ns' in Eqs. (3) and (4) stands for any of the following three types of combinations of (parton or fragmentation) quark distributions,

$$
f_{i k}^{ \pm}=q_{i} \pm \bar{q}_{i}-\left(q_{k} \pm \bar{q}_{k}\right), \quad f^{\mathrm{v}}=\sum_{r=1}^{n_{f}}\left(q_{r}-\bar{q}_{r}\right)
$$

where $n_{f}$ denotes the number of active (effectively massless) flavours. As detailed below, we have obtained the so far unknown time-like NNLO splitting functions $P_{\sigma=1}^{(2) \mathrm{ns}}(x)$ in Eq. (4).

As already indicated in Eq. (4), the space-like and time-like non-singlet splitting functions are identical at the leading order (LO) [8], a fact known as the Gribov-Lipatov relation. This relation does not hold beyond LO in the usual $\overline{\mathrm{MS}}$ scheme adopted also in this letter. However, the space-like and time-like cases are related by an analytic continuation in $x$, as shown in detailed diagrammatic analyses [5,9] at order $\alpha_{\mathrm{s}}^{2}$, see also Refs. [10,11]. Moreover, another approach relating the non-singlet splitting functions has been proposed in Ref. [12]. Hence it should be possible to derive time-like quantities from the space-like results computed to order $\alpha_{\mathrm{s}}^{3}$ in Refs. [13-15]. 
We start the analytic continuation from the unrenormalized (and unfactorized) partonic transverse structure function $F_{1}^{\mathrm{b}}$ in deep-inelastic scattering, $\gamma^{*} q \rightarrow X$ (and correspondingly for $F_{L}$ and $F_{3} \rightarrow F_{A}$ ), calculated in dimensional regularization with $D=4-2 \varepsilon$ and the scale $\mu$ [13-15],

$$
F_{1}^{\mathrm{b}}\left(a_{\mathrm{s}}^{\mathrm{b}}, Q^{2}\right)=\delta(1-x)+\sum_{l=1}^{\infty}\left(a_{\mathrm{s}}^{\mathrm{b}}\right)^{l}\left(\frac{Q^{2}}{\mu^{2}}\right)^{-l \varepsilon} F_{1, l}^{\mathrm{b}}
$$

The bare and renormalized coupling $\alpha_{\mathrm{s}}^{\mathrm{b}}$ and $\alpha_{\mathrm{s}}$ are related by (recall $a_{\mathrm{s}} \equiv \alpha_{\mathrm{s}} /(4 \pi)$ )

$$
a_{\mathrm{s}}^{\mathrm{b}}=a_{\mathrm{s}}-\frac{\beta_{0}}{\varepsilon} a_{\mathrm{s}}^{2}+\left(\frac{\beta_{0}^{2}}{\varepsilon^{2}}-\frac{\beta_{1}}{2 \varepsilon}\right) a_{\mathrm{s}}^{3}+\ldots
$$

with $\beta_{0}=11 / 3 C_{A}-2 / 3 n_{f}$ etc. The expansion coefficients in Eq. (6) are then decomposed into form-factor $\left(\mathcal{F}_{l}\right)$ and real-emission $\left(\mathcal{R}_{l}\right.$, defined analogous to Eq. [6]) contributions [16]

$$
\begin{aligned}
F_{1,1}^{\mathrm{b}} & =2 \mathcal{F}_{1} \delta(1-x)+\mathcal{R}_{1} \\
F_{1,2}^{\mathrm{b}} & =2 \mathcal{F}_{2} \delta(1-x)+\left(\mathcal{F}_{1}\right)^{2} \delta(1-x)+2 \mathcal{F}_{1} \mathcal{R}_{1}+\mathcal{R}_{2} \\
F_{1,3}^{\mathrm{b}} & =2 \mathcal{F}_{3} \delta(1-x)+2 \mathcal{F}_{1} \mathcal{F}_{2} \delta(1-x)+\left(2 \mathcal{F}_{2}+\left(\mathcal{F}_{1}\right)^{2}\right) \mathcal{R}_{1}+2 \mathcal{F}_{1} \mathcal{R}_{2}+\mathcal{R}_{3} .
\end{aligned}
$$

The analytic continuation of the form factor to the time-like case is known. The $x$-dependent functions $\mathcal{R}_{l}$ are continued from $x$ to $1 / x[5,9]$, taking into account the (complex) continuation of $q^{2}$ (see Eq. (4.1) of Ref. [16]) and the additional prefactor $x^{1-2 \varepsilon}$ originating from the phase space of the detected parton in the time-like case [3]. Practically this continuation has been performed using routines for harmonic polylogarithms [17, 18] implemented in FORM [19]. The only subtle point in the analytic continuations is the treatment of logarithmic singularities for $x \rightarrow 1$, cf. Ref. [9], starting with

$$
\ln (1-x) \rightarrow \ln (1-x)-\ln x+i \pi
$$

Finally the bare transverse fragmentation function $F_{T}^{\mathrm{b}}$ is re-assembled analogous to Eq. (8), keeping the real parts of the continued $\mathcal{R}_{l}$ only, and the time-like non-singlet splitting functions and coefficient functions can be read off iteratively from the non-singlet mass factorization formula

$$
\begin{aligned}
F_{T, 1}= & -\varepsilon^{-1} P^{(0)}+c_{T}^{(1)}+\varepsilon a_{T}^{(1)}+\varepsilon^{2} b_{T}^{(1)}+\varepsilon^{3} d_{T}^{(1)}+\ldots \\
F_{T, 2}= & \frac{1}{2 \varepsilon^{2}} P^{(0)}\left(P^{(0)}+\beta_{0}\right)-\frac{1}{2 \varepsilon}\left[P_{\sigma=1}^{(1)}+2 P^{(0)} c_{T}^{(1)}\right]+c_{T}^{(2)}-P^{(0)} a_{T}^{(1)}+\varepsilon\left[a_{T}^{(2)}-P^{(0)} b_{T}^{(1)}\right]+\ldots \\
F_{T, 3}= & -\frac{1}{6 \varepsilon^{3}} P^{(0)}\left(P^{(0)}+\beta_{0}\right)\left(P^{(0)}+2 \beta_{0}\right)+\frac{1}{6 \varepsilon^{2}}\left[P_{\sigma=1}^{(1)}\left(3 P^{(0)}+2 \beta_{0}\right)+P^{(0)}\left(3 P^{(0)} c_{T}^{(1)}\right.\right. \\
& \left.\left.+3 \beta_{0} c_{T}^{(1)}+2 \beta_{1}\right)\right]-\frac{1}{6 \varepsilon}\left[2 P_{\sigma=1}^{(2)}+3 P_{\sigma=1}^{(1)} c_{T}^{(1)}+P^{(0)}\left(6 c_{T}^{(2)}-3 P^{(0)} a_{T}^{(1)}-3 \beta_{0} a_{T}^{(1)}\right)\right]+\ldots
\end{aligned}
$$

where the expansion coefficients $F_{T, l}$ now refer to an expansion in the renormalized coupling at the scale $Q^{2} \equiv q^{2}$. The products of the $x$-dependent (generalized) functions in Eq. (10) are to be read as Mellin convolutions or, more conveniently, as products in Mellin- $N$ space, employing routines for harmonic sums and their inverse Mellin transform back to $x$-space [18-20]. 
Unlike the diagrammatic treatment of Refs. [5,9] — which cannot be emulated at order $\alpha_{\mathrm{s}}^{3}$ using the only available space-like calculation based on the optical theorem [13-15] — the above procedure is not entirely rigorous, as Eq. [8] does not represent a full decomposition according to the number of emitted partons. This would be required, for instance, in a subtraction formalism for exclusive observables at NNLO [21] and beyond. In Eq. (8) the real functions $\mathcal{R}_{n \geq 2}$ do not only collect, e.g., $n$-gluon tree-level amplitudes, but also combinations of real emission and virtual corrections. Especially, starting at order $\alpha_{\mathrm{s}}^{3}$, the decomposition Eq. [8] includes overlapping divergences from triple unresolved configurations when two particles become soft and one is collinear. Thus one has to be prepared for some problem in the abelian $\left(C_{F}^{3}\right)$ piece related to $\pi^{2}$ contributions originating from phase space integrations over unresolved regions.

At the second order, however, the above procedure works perfectly at least for the terms in the $\varepsilon$-expansion written down in the second line of Eq. (10), thus including the previously unknown $\varepsilon$-coefficients $a_{a}^{(2)}(x), a=T, L, A$, which we will not write out here for brevity. We have verified this by comparing to a direct calculation, to be presented elsewhere, of $e^{+} e^{-} \rightarrow \gamma, Z \rightarrow h+X$ to this accuracy in $\varepsilon$ using the approach of Ref. [22]. Especially, we also re-derive the $O\left(a_{\mathrm{s}}^{2}\right)$ coefficient functions $c_{a}^{(2)}(x)$ which so far were only calculated in Refs. [2-4]. The differences between the time-like non-singlet coefficient functions for Eq. (1) and the corresponding (by the structure of the respective hadronic tensors) quantities in deep-inelastic scattering read

$$
\begin{aligned}
c_{T, \mathrm{~ns}}^{(2)} & (x)-c_{1, \mathrm{~ns}}^{(2)}(x)= \\
+ & C_{F}^{2}\left(72-8 \zeta_{2}+176 / 3 \mathrm{H}_{0}-48 \mathrm{H}_{0,0}+8 \mathrm{H}_{2}-72 \mathrm{H}_{1,0}+(1+x)\left[-493 / 6+12 \zeta_{3}\right.\right. \\
& +10 \zeta_{2}-155 / 6 \mathrm{H}_{0}+16 \mathrm{H}_{0} \zeta_{2}-6 \mathrm{H}_{0,0}+12 \mathrm{H}_{0,0,0}-20 \mathrm{H}_{3}-10 \mathrm{H}_{2}-4 \mathrm{H}_{2,0}-29 \mathrm{H}_{1} \\
& \left.+42 \mathrm{H}_{1,0}-12 \mathrm{H}_{1,1}\right]+p_{\mathrm{qq}}(x)\left[-84 \zeta_{3}-106 / 3 \zeta_{2}+389 / 6 \mathrm{H}_{0}-44 \mathrm{H}_{0} \zeta_{2}\right. \\
& +196 / 3 \mathrm{H}_{0,0}-24 \mathrm{H}_{0,0,0}+36 \mathrm{H}_{3}+62 \mathrm{H}_{2}-20 \mathrm{H}_{2,0}+48 \mathrm{H}_{2,1}-40 \mathrm{H}_{1} \zeta_{2}+134 / 3 \mathrm{H}_{1,0} \\
& \left.\left.+32 \mathrm{H}_{1,0,0}+56 \mathrm{H}_{1,2}+40 \mathrm{H}_{1,1,0}\right]+\delta(1-x)\left[608 / 3 \zeta_{2}-24 \zeta_{2}^{2}\right]\right) \\
+ & C_{F}\left(C_{A}-2 C_{F}\right)\left(-76 / 3 x \mathrm{H}_{0}+(1+x)\left[-215 / 6+49 / 6 \mathrm{H}_{0}-9 \mathrm{H}_{1}\right]+p_{\mathrm{qq}}(x)\left[12 \zeta_{3}\right.\right. \\
& -44 / 3 \zeta_{2}+445 / 6 \mathrm{H}_{0}-20 \mathrm{H}_{0} \zeta_{2}+44 / 3 \mathrm{H}_{0,0}+24 \mathrm{H}_{0,0,0}+4 \mathrm{H}_{3}+22 \mathrm{H}_{2}-4 \mathrm{H}_{2,0} \\
& \left.-16 \mathrm{H}_{1} \zeta_{2}+22 / 3 \mathrm{H}_{1,0}+8 \mathrm{H}_{1,2}-8 \mathrm{H}_{1,1,0}\right]+p_{\mathrm{qq}}(-x)\left[32 \mathrm{H}_{-1,0,0}+8 \mathrm{H}_{0} \zeta_{2}+16 \mathrm{H}_{-2,0}\right. \\
& \left.\left.-24 \mathrm{H}_{0,0,0}\right]+\delta(1-x)\left[466 / 3 \zeta_{2}-24 \zeta_{2}^{2}\right]\right) \\
+ & C_{F} n_{f}\left(4 / 3 x \mathrm{H}_{0}+(1+x)\left[19 / 3+1 / 3 \mathrm{H}_{0}+2 \mathrm{H}_{1}\right]+p_{\mathrm{qq}}(x)\left[8 / 3 \zeta_{2}-35 / 3 \mathrm{H}_{0}\right.\right. \\
& \left.\left.-8 / 3 \mathrm{H}_{0,0}-4 \mathrm{H}_{2}-4 / 3 \mathrm{H}_{1,0}\right]+\delta(1-x)\left[-76 / 3 \zeta_{2}\right]\right) \\
c_{L, \mathrm{~ns}} & \left.(x)\right|_{e^{+} e^{-}}-\left.c_{L, \mathrm{~ns}}^{(2)}(x)\right|_{e p}= \\
+ & C_{F}^{2}\left(583 / 9-860 / 9 x-(14 / 3+152 / 3 x) \mathrm{H}_{0}-(12+16 x) \mathrm{H}_{0,0}+(74 / 3-112 / 3 x) \mathrm{H}_{1}\right. \\
& \left.-16(1+x) \mathrm{H}_{1,0}+8(1-2 x) \mathrm{H}_{1,1}+4(1+6 x)\left[\zeta_{2}-\mathrm{H}_{2}\right]\right) \\
+ & C_{F}\left(C_{A}-2 C_{F}\right)\left(2317 / 45+8 / 5 x^{-1}-3752 / 45 x+32 / 5 x^{2}+32 x \zeta_{3}+\left(40+8 / 5 x^{-2}\right) \mathrm{H}_{-1,0}\right.
\end{aligned}
$$




$$
\begin{aligned}
& -1 / 5\left(98 / 3+8 x^{-1}+728 / 3 x-32 x^{2}\right) \mathrm{H}_{0}+16 \mathrm{H}_{0,-1,0}+32\left(x-1 / 5 x^{3}\right)\left[\zeta_{2}+\mathrm{H}_{-1,0}-\mathrm{H}_{0,0}\right] \\
& \left.-8(1+2 x)\left[\mathrm{H}_{-1} \zeta_{2}+2 \mathrm{H}_{-1,-1,0}-\mathrm{H}_{-1,0,0}\right]+(1-2 x)\left[46 / 3 \mathrm{H}_{1}-8 \mathrm{H}_{1} \zeta_{2}+8 \mathrm{H}_{1,0,0}\right]\right) \\
& +C_{F} n_{f}\left(-74 / 9+112 / 9 x+4 / 3(1+4 x) \mathrm{H}_{0}-4 / 3(1-2 x) \mathrm{H}_{1}\right) \\
c_{A, \mathrm{~ns}}^{(2)}(x)-c_{3, \mathrm{~ns}}^{(2)}(x)=c_{T, \mathrm{~ns}}^{(2)}(x)-c_{1, \mathrm{~ns}}^{(2)}(x) & +C_{F}^{2}\left((1-x)\left[-16 \zeta_{2}+46 \mathrm{H}_{0}+20 \mathrm{H}_{0,0}+16 \mathrm{H}_{2}+24 \mathrm{H}_{1,0}\right]\right)+C_{F} n_{f}\left(-4(1-x) \mathrm{H}_{0}\right) \\
+ & C_{F}\left(C_{A}-2 C_{F}\right)\left(p_{\mathrm{qq}}(-x)\left[-64 \mathrm{H}_{-1,0,0}-16 \mathrm{H}_{0} \zeta_{2}-32 \mathrm{H}_{-2,0}+48 \mathrm{H}_{0,0,0}\right]\right. \\
& \left.+54(1-x) \mathrm{H}_{0}+32(1+x) \mathrm{H}_{0,0}\right) .
\end{aligned}
$$

Here our notation for the harmonic polylogarithms $H_{m_{1}, \ldots, m_{w}}(x), m_{j}=0, \pm 1$ follows Ref. [17]. Furthermore we have employed the short-hand notation

$$
H_{\underbrace{0, \ldots, 0}_{m}}, \pm 1, \underbrace{0, \ldots, 0}_{n}, \pm 1, \ldots(x)=H_{ \pm(m+1), \pm(n+1), \ldots}(x)
$$

suppressed the argument of the polylogarithms, and used the function $p_{\mathrm{qq}}(x)=2(1-x)^{-1}-1-x$. The divergences for $x \rightarrow 1$ in Eq. (11) are to be read as plus-distributions.

Eqs. (11) - (13) agree with the results in Refs. [3,4] up to a few (presumably typographical) errors in those articles. Specifically, for $c_{T}^{(2)}(z)$ the term $-3 C_{F}^{2}(1+z) \ln ^{2} z$ in Eq. (A.6) of Ref. [3] has to be replaced by $-3 C_{F}^{2}(1+z) \ln ^{3} z$, and the contribution $24\left(C_{F}^{2}-C_{A} C_{F} / 2\right) \ln z /\left(5 z^{2}\right)$ in Eq. (A.8) by $24\left(C_{F}^{2}-C_{A} C_{F} / 2\right) \ln z /(5 z)$. The argument of $S_{1,2}$ should read $-z$ instead of $1-z$ in the first term of Eq. (A.15) for $c_{L}^{(2)}(z)$. Finally, in Eq. (17) of Ref. [4] for $c_{A}^{(2)}(z)$ the term $24 C_{F}^{2} \ln z /\left(5 z^{2}\right)$ has to be replaced by $24 C_{F}^{2} \ln z /(5 z)$. We have also re-calculated the second-order gluon and pure-singlet coefficient functions for $c_{T}$ and $c_{L}$, finding complete agreement with Ref. [4].

We now turn to the corresponding NNLO (third-order) splitting functions $P_{\sigma=1}^{(2) n s}$ in Eq. (4). Using the same notation as above these functions are given by

$$
\begin{aligned}
\delta P^{(2)}+ & (x) \equiv P_{\sigma=1}^{(2)+}(x)-P_{\sigma=-1}^{(2)+}(x)= \\
+ & 16 C_{F}^{3}\left(p _ { \mathrm { qq } } ( x ) \left[311 / 24 \mathrm{H}_{0}+4 / 3 \mathrm{H}_{0} \zeta_{2}-169 / 9 \mathrm{H}_{0,0}+8 \mathrm{H}_{0,0} \zeta_{2}-22 \mathrm{H}_{0,0,0}\right.\right. \\
& \left.-268 / 9 \mathrm{H}_{1,0}+8 \mathrm{H}_{1,0} \zeta_{2}-44 / 3 \mathrm{H}_{1,0,0}-268 / 9 \mathrm{H}_{2}+8 \mathrm{H}_{2} \zeta_{2}-44 / 3 \mathrm{H}_{2,0}-44 / 3 \mathrm{H}_{3}\right] \\
& +(1+x)\left[-4 \mathrm{H}_{0,0} \zeta_{2}+25 / 2 \mathrm{H}_{0,0,0}+\mathrm{H}_{2,0}+2 \mathrm{H}_{3}\right]-(1-x)\left[325 / 18 \mathrm{H}_{0}+50 / 3 \mathrm{H}_{1,0}\right. \\
& \left.\left.+50 / 3 \mathrm{H}_{2}\right]+(3-5 x) \mathrm{H}_{0} \zeta_{2}-(173 / 18-691 / 18 x) \mathrm{H}_{0,0}\right) \\
+ & 16 C_{F}^{2}\left(C_{A}-2 C_{F}\right)\left(p _ { \mathrm { qq } } ( x ) \left[151 / 24 \mathrm{H}_{0}+\mathrm{H}_{0} \zeta_{3}+13 / 6 \mathrm{H}_{0} \zeta_{2}-169 / 18 \mathrm{H}_{0,0}+8 \mathrm{H}_{0,0} \zeta_{2}\right.\right. \\
& -13 / 2 \mathrm{H}_{0,0,0}-8 \mathrm{H}_{0,0,0,0}-134 / 9 \mathrm{H}_{1,0}+4 \mathrm{H}_{1,0} \zeta_{2}-22 / 3 \mathrm{H}_{1,0,0}-6 \mathrm{H}_{1,0,0,0}-134 / 9 \mathrm{H}_{2} \\
& \left.+4 \mathrm{H}_{2} \zeta_{2}-22 / 3 \mathrm{H}_{2,0}-2 \mathrm{H}_{2,0,0}-22 / 3 \mathrm{H}_{3}-2 \mathrm{H}_{3,0}-6 \mathrm{H}_{4}\right]+p_{\mathrm{qq}}(-x)\left[-8 \mathrm{H}_{-3,0}\right. \\
& +8 \mathrm{H}_{-2} \zeta_{2}+8 \mathrm{H}_{-2,-1,0}+3 \mathrm{H}_{-2,0}-14 \mathrm{H}_{-2,0,0}-4 \mathrm{H}_{-2,2}+8 \mathrm{H}_{-1,-2,0}+16 \mathrm{H}_{-1,-1,0,0} \\
& +8 \mathrm{H}_{-1,0} \zeta_{2}+6 \mathrm{H}_{-1,0,0}-18 \mathrm{H}_{-1,0,0,0}-4 \mathrm{H}_{-1,2,0}-8 \mathrm{H}_{-1,3}-7 \mathrm{H}_{0} \zeta_{3}+3 / 2 \mathrm{H}_{0} \zeta_{2}
\end{aligned}
$$




$$
\begin{aligned}
& \left.-8 \mathrm{H}_{0,0} \zeta_{2}-9 / 2 \mathrm{H}_{0,0,0}+8 \mathrm{H}_{0,0,0,0}+2 \mathrm{H}_{3,0}+6 \mathrm{H}_{4}\right]-(1+x)\left[4 \mathrm{H}_{-2,0}+8 \mathrm{H}_{-1,0,0}\right] \\
& +(1-x)\left[4 \mathrm{H}_{-3,0}+4 \mathrm{H}_{-2,0,0}-88 / 9 \mathrm{H}_{0}+3 \mathrm{H}_{0} \zeta_{3}-28 / 3 \mathrm{H}_{1,0}-28 / 3 \mathrm{H}_{2}\right]-4 x \mathrm{H}_{0} \zeta_{2} \\
& \left.-(50 / 9-184 / 9 x) \mathrm{H}_{0,0}-4 x \mathrm{H}_{0,0} \zeta_{2}+(11 / 2+35 / 2 x) \mathrm{H}_{0,0,0}+8 x \mathrm{H}_{0,0,0,0}\right) \\
& +16 C_{F}^{2} n_{f}\left(p _ { \mathrm { qq } } ( x ) \left[-11 / 12 \mathrm{H}_{0}-2 / 3 \mathrm{H}_{0} \zeta_{2}+11 / 9 \mathrm{H}_{0,0}+2 \mathrm{H}_{0,0,0}+20 / 9 \mathrm{H}_{1,0}\right.\right. \\
& \left.+4 / 3 \mathrm{H}_{1,0,0}+20 / 9 \mathrm{H}_{2}+4 / 3 \mathrm{H}_{2,0}+4 / 3 \mathrm{H}_{3}\right]-(1+x) \mathrm{H}_{0,0,0}+(1-x)\left[13 / 9 \mathrm{H}_{0}\right. \\
& \left.\left.+4 / 3 \mathrm{H}_{1,0}+4 / 3 \mathrm{H}_{2}\right]+(8 / 9-28 / 9 x) \mathrm{H}_{0,0}\right),
\end{aligned}
$$

and

$$
\begin{aligned}
& \delta P^{(2) \xi}(x)-\delta P^{(2)+}(x) \equiv \\
& +16 C_{F}^{2}\left(C_{A}-2 C_{F}\right)\left(p _ { \mathrm { qq } } ( - x ) \left[16 \mathrm{H}_{-3,0}-16 \mathrm{H}_{-2} \zeta_{2}-16 \mathrm{H}_{-2,-1,0}-6 \mathrm{H}_{-2,0}+28 \mathrm{H}_{-2,0,0}\right.\right. \\
& +8 \mathrm{H}_{-2,2}-16 \mathrm{H}_{-1,-2,0}-32 \mathrm{H}_{-1,-1,0,0}-16 \mathrm{H}_{-1,0} \zeta_{2}-12 \mathrm{H}_{-1,0,0}+36 \mathrm{H}_{-1,0,0,0}+8 \mathrm{H}_{-1,2,0} \\
& \left.+16 \mathrm{H}_{-1,3}+14 \mathrm{H}_{0} \zeta_{3}-3 \mathrm{H}_{0} \zeta_{2}+16 \mathrm{H}_{0,0} \zeta_{2}+9 \mathrm{H}_{0,0,0}-16 \mathrm{H}_{0,0,0,0}-4 \mathrm{H}_{3,0}-12 \mathrm{H}_{4}\right] \\
& +(1+x)\left[8 \mathrm{H}_{-2,0}+16 \mathrm{H}_{-1,0,0}+8 \mathrm{H}_{0} \zeta_{2}-4 \mathrm{H}_{2,0}-8 \mathrm{H}_{3}\right]-(1-x)\left[8 \mathrm{H}_{-3,0}+8 \mathrm{H}_{-2,0,0}+10 \mathrm{H}_{0}\right. \\
& \left.\left.+6 \mathrm{H}_{0} \zeta_{3}+4 \mathrm{H}_{0,0} \zeta_{2}-8 \mathrm{H}_{0,0,0,0}+8 \mathrm{H}_{1,0}+8 \mathrm{H}_{2}\right]-(10-6 x) \mathrm{H}_{0,0}-(12+24 x) \mathrm{H}_{0,0,0}\right)
\end{aligned}
$$

for both $\xi=-$ and $\xi=$ v. Eq. (15) is, in fact, not quite the result of the analytic continuation as described above, which returns a different coefficient for the term $C_{F}^{3} p_{\mathrm{qq}}(x) \mathrm{H}_{0,0} \zeta_{2}$ in the first line. We have corrected this term by imposing the correct (vanishing) first moment of $P_{\sigma=1}^{(2)-}$. As it is conceivable that the present form of the analytic continuation leads to other problems not affecting the first moment - recall the discussion in the paragraph below Eq. (10) - we obviously need a second, independent confirmation of our new results (15) and (16).

For this purpose we adopt the approach of Dokshitzer, Marchesini and Salam [12] (see also Appendix B1 of Ref. [23]), where Eq. (3) is rewritten as ${ }^{1}$

$$
\frac{d}{d \ln Q^{2}} f_{\sigma}^{\mathrm{ns}}\left(x, Q^{2}\right)=\int_{x}^{1} \frac{d z}{z} P_{\mathrm{univ}}^{\mathrm{ns}}\left(z, \alpha_{\mathrm{s}}\left(Q^{2}\right)\right) f_{\sigma}^{\mathrm{ns}}\left(\frac{x}{z}, z^{\sigma} Q^{2}\right)
$$

and the modified splitting functions $P_{\text {univ }}^{\text {ns }}$ are postulated to be identical for the time-like and spacelike cases. Working out the perturbative expansion of the integrand along the lines of Ref. [12] one arrives at a successful 'postdiction' for the NLO difference $P_{\sigma=1}^{(1) \mathrm{ns}}(x)-P_{\sigma=-1}^{(1) \mathrm{ns}}(x)$ of Refs. [5, 7] (see Eq. (4) of Ref. [12]) and the new NNLO prediction ( $\xi=+,-, \mathrm{v}$, recall Eq. (5))

$$
\delta P^{(2) \xi}(x)=2\left\{\left[\ln x \cdot \widetilde{P}^{(1) \xi}\right] \otimes P^{(0)}+\left[\ln x \cdot P^{(0)}\right] \otimes \widetilde{P}^{(1) \xi}\right\}
$$

with $\otimes$ denoting the Mellin convolution (cf. Eqs. (2) and (3)) and

$$
2 \widetilde{P}^{(n) \xi}(x)=P_{\sigma=1}^{(n) \xi}(x)+P_{\sigma=-1}^{(n) \xi}(x) .
$$

\footnotetext{
${ }^{1}$ Ref. [12] also includes a shift in the argument of $\alpha_{s}$ in Eq. 17 which is irrelevant for our purpose of relating the time-like and space-like results. Note also that the notation for the $\alpha_{\mathrm{s}}$ expansion in Ref. [12] differs from Eq. (4).
} 
The evaluation of Eq. (18), again performing the convolutions via a transformation to Mellin- $N$ space, yields exactly Eqs. (15) and (16), thus providing both the desired confirmation of these results and further evidence supporting the ansatz (17).

Consequently, it is possible to make even a prediction for the fourth-order $\left(\mathrm{N}^{3} \mathrm{LO}\right)$ difference $\delta P^{(3) \xi}$ of the (both unknown) time-like and space-like non-singlet splitting functions on this basis. Using the notation of Eq. (18) together with $A^{\otimes 2} \equiv A \otimes A$ etc, this prediction reads

$$
\begin{aligned}
\delta P^{(3) \xi}(x)= & 2\left\{\left[\ln x \cdot \widetilde{P}^{(2) \xi}\right] \otimes P^{(0)}+\left[\ln x \cdot P^{(0)}\right] \otimes \widetilde{P}^{(2) \xi}+\left[\ln x \cdot \widetilde{P}^{(1) \xi}\right] \otimes \widetilde{P}^{(1) \xi}\right\} \\
& -2 P^{(0)} \otimes\left[\ln x \cdot P^{(0)}\right]^{\otimes 3}-4\left[P^{(0)}\right]^{\otimes 2} \otimes\left[\ln x \cdot P^{(0)}\right] \otimes\left[\ln ^{2} x \cdot P^{(0)}\right] \\
& -2 / 3\left[P^{(0)}\right]^{\otimes 3} \otimes\left[\ln ^{3} x \cdot P^{(0)}\right] .
\end{aligned}
$$

For brevity we refrain from writing out the resulting explicit expressions which are, of course, much more lengthy than Eq. (15). We expect that a first check of Eq. (20), or rather its first line (which dominates the large- $x$ behaviour, cf. Ref. [12]), will be obtained via next-to-leading order calculations in the large- $n_{f}$ expansion, generalizing the leading- $n_{f}$ result of Ref. [24]. The corresponding contribution to Eq. (20) is identical for all three non-singlet cases and reads

$$
\begin{aligned}
& \left.\delta P^{(3), \mathrm{ns}}(x)\right|_{n_{f}^{2}} \equiv P_{\sigma=1}^{(3), \mathrm{ns}}(x)-P_{\sigma=-1}^{(3), \mathrm{ns}}(x)= \\
& 16 / 81 C_{F}^{2} n_{f}^{2}\left(p _ { \mathrm { qq } } ( x ) \left[-\left(159 / 4-120 \zeta_{2}+36 \zeta_{3}\right) \mathrm{H}_{0}+\left(23+72 \zeta_{2}\right) \mathrm{H}_{0,0}-279 \mathrm{H}_{0,0,0}\right.\right. \\
& \quad-216 \mathrm{H}_{0,0,0,0}-76\left(\mathrm{H}_{1,0}+\mathrm{H}_{2}\right)-240\left(\mathrm{H}_{1,0,0}+\mathrm{H}_{2,0}+\mathrm{H}_{3}\right)-108\left(\mathrm{H}_{1,0,0,0}+\mathrm{H}_{2,0,0}\right. \\
& \left.\left.\quad+\mathrm{H}_{3,0}+\mathrm{H}_{4}\right)\right]+(1-x)\left[-\left(260-72 \zeta_{2}\right) \mathrm{H}_{0}-276\left(\mathrm{H}_{1,0}+\mathrm{H}_{2}\right)-144\left(\mathrm{H}_{1,0,0}+\mathrm{H}_{2,0}\right.\right. \\
& \left.\left.\left.\quad+\mathrm{H}_{3}\right)\right]+(1+x) 108 \mathrm{H}_{0,0,0,0}-(466-398 x) \mathrm{H}_{0,0}-(90-450 x) \mathrm{H}_{0,0,0}\right) .
\end{aligned}
$$

The $a_{\mathrm{s}}^{n} C_{F}^{2} n_{f}^{n-2}$ contributions dominating $\delta P^{(n-1), \text { ns }}$ in the large- $n_{f}$ limit are given to all higher orders $n$ by a straightforward generalization of Eq. (18) and the first line of Eq. (20).

Returning to the NNLO coefficient-function and splitting-function differences 111 - (16), we note that these functions include harmonic polylogarithms up to the same weights as the corresponding space-like results [13-15,18], with the interesting exception that weight-4 functions enter Eq. (15) only for the $\mathrm{SU}\left(n_{c}\right)$ group-factor combination $C_{A}-2 C_{F}$ suppressed as $1 / n_{c}$ in the limit of a large number of colours $n_{c}$. Except for the longitudinal coefficient function $c_{L, \mathrm{~ns}}$, the differences between the time-like and space-like quantities are parametrically suppressed in the large- $x$ limit, since the logarithmically enhanced soft-emission contributions to $\mathcal{R}_{l}$ in Eq. (8) are, as they have to be, invariant under the analytic continuation. Specifically, the (identical) leading large- $x$ contributions for $c_{T, \mathrm{~ns}}^{(2)}-c_{1, \mathrm{~ns}}^{(2)}$ and $c_{A, \mathrm{~ns}}^{(2)}-c_{3, \mathrm{~ns}}^{(2)}$ contain plus-distributions only up to $\left[(1-x)^{-1} \ln (1-x)\right]_{+}$ (all proportional to $\pi^{2}$-terms arising from the analytic continuation of the form factor), and those for the splitting-function differences read,

$$
\delta P^{(2), \mathrm{ns}}(x \rightarrow 1)=-4 A_{\mathrm{q}}^{(1)} A_{\mathrm{q}}^{(2)} \ln (1-x)+O(1),
$$

as predicted in Ref. [12], where $A_{\mathrm{q}}^{(n)}$ are the coefficients of $a_{\mathrm{s}}^{n}[1-x]_{+}^{-1}$ in Eq. (4), cf. Ref. [13]. The leading small- $x$ terms, on the other hand, differ between the space-like and time-like cases. 
The numerical impact of the second-order contributions to the time-like coefficient functions $c_{a}^{(2)}(x), a=T, L, A$, has been discussed in some detail already in Refs. [2-4]. Here we confine ourselves to the transverse fragmentation function $F_{T}$, the largest contribution to the right-hand-side of Eq. (1), see Ref. [1]. In Fig. 11 we compare the corresponding non-singlet coefficient function $c_{T, \mathrm{~ns}}$ to its counterpart $c_{1, \mathrm{~ns}}$ in deep-inelastic scattering. In order to facilitate a direct comparison, the same schematic shape has been used for the non-singlet fragmentation distributions and parton distributions. As obvious from the figure, the higher-order corrections for $c_{T}$ are in general considerably larger than those for $c_{1}$. Nevertheless the second-order term changes the NLO results, under the conditions of Fig. 1. by $5 \%$ or less from $x \simeq 0.55$ down to very small values of $x$.

The pattern is quite different for the non-singlet splitting functions illustrated, in a similar manner but at a lower scale, in Fig. 2 In $N$-space, for example, the ratio $\delta P^{\text {ns }} /\left(P_{\sigma=-1}^{\text {ns }}-P_{\mathrm{LO}}^{\text {ns }}\right)$ quickly decreases with increasing $N$, at NNLO from about $1 / 2$ at $N=2$ to about $1 / 6$ at $N=8$. Consequently the total time-like splitting functions $P_{\sigma=1}^{\text {ns }}$ is only mildly enhanced, e.g., by $8 \%$ and $2 \%$ for these two values of $N$ and $\alpha_{\mathrm{s}}=0.2$, with respect to their space-like counterparts $P_{\sigma=-1}^{\mathrm{ns}}$ discussed in detail in Ref. [13]. As shown in the right part of Fig. [2 the small- $x$ scaling violations of the non-singlet fragmentation distributions are weaker than those of the parton distributions. For the chosen input distribution this reduction increases, in a perturbatively stable manner, from about $10 \%$ at $x=10^{-2}$ to about $30 \%$ at $x=10^{-4}$.

To summarize, we have re-derived the $O\left(\alpha_{\mathrm{s}}^{2}\right)$ coefficient functions [2-4] for the inclusive production of single hadrons in $e^{+} e^{-}$annihilation [1] and obtained, for the first time, the corresponding third-order splitting functions for the flavour non-singlet fragmentation distributions. Our derivation of the latter quantities rests on relations between the time-like and space-like cases, see especially Refs. $[5,7,9,12]$, and the third-order calculation of deep-inelastic scattering of Ref. [13-15]. We expect that a further study of these relations, backed up by fixed Mellin- $N$ calculations along the lines of Ref. [22], will facilitate an extension of our derivation to the NNLO flavour-singlet splitting functions and, at least for $F_{L}$, the $O\left(\alpha_{\mathrm{s}}^{3}\right)$ coefficient functions.

Once this step has been taken, the way is open for full NNLO analyses, e.g., along the lines of Ref. [25], of high-precision data on $e^{+} e^{-} \rightarrow h+X$ from LEP and a future International Linear Collider. Due to the universality of the splitting functions, our results also represents a first step towards NNLO analyses of high- $p_{T}$ hadron production in $e p$ and $p p$ collisions, where very large NLO corrections strongly suggest sizeable higher-order contributions, see Refs. [26-28] and [29-31], respectively. Another application concerns the $b$-quark spectrum in top decays (see Ref. [32]) where, after the calculations of Refs. [33,34], the third-order time-like splitting functions will facilitate a complete NNLO treatment in the framework of perturbative fragmentation.

FORM and FORTRAN files of our results can be obtained from http://arXiv.org by downloading the source of this article. Furthermore they are available from the authors upon request.

Acknowledgments: We are grateful to L. Dixon, P. Uwer and W. Vogelsang for useful discussions. Our numerical results have been computed using the FORTRAN package of Ref. [35]. A.M. acknowledges support by the Alexander von Humboldt Foundation. The work of S.M. has been supported in part by the Helmholtz Gemeinschaft under contract VH-NG-105. 

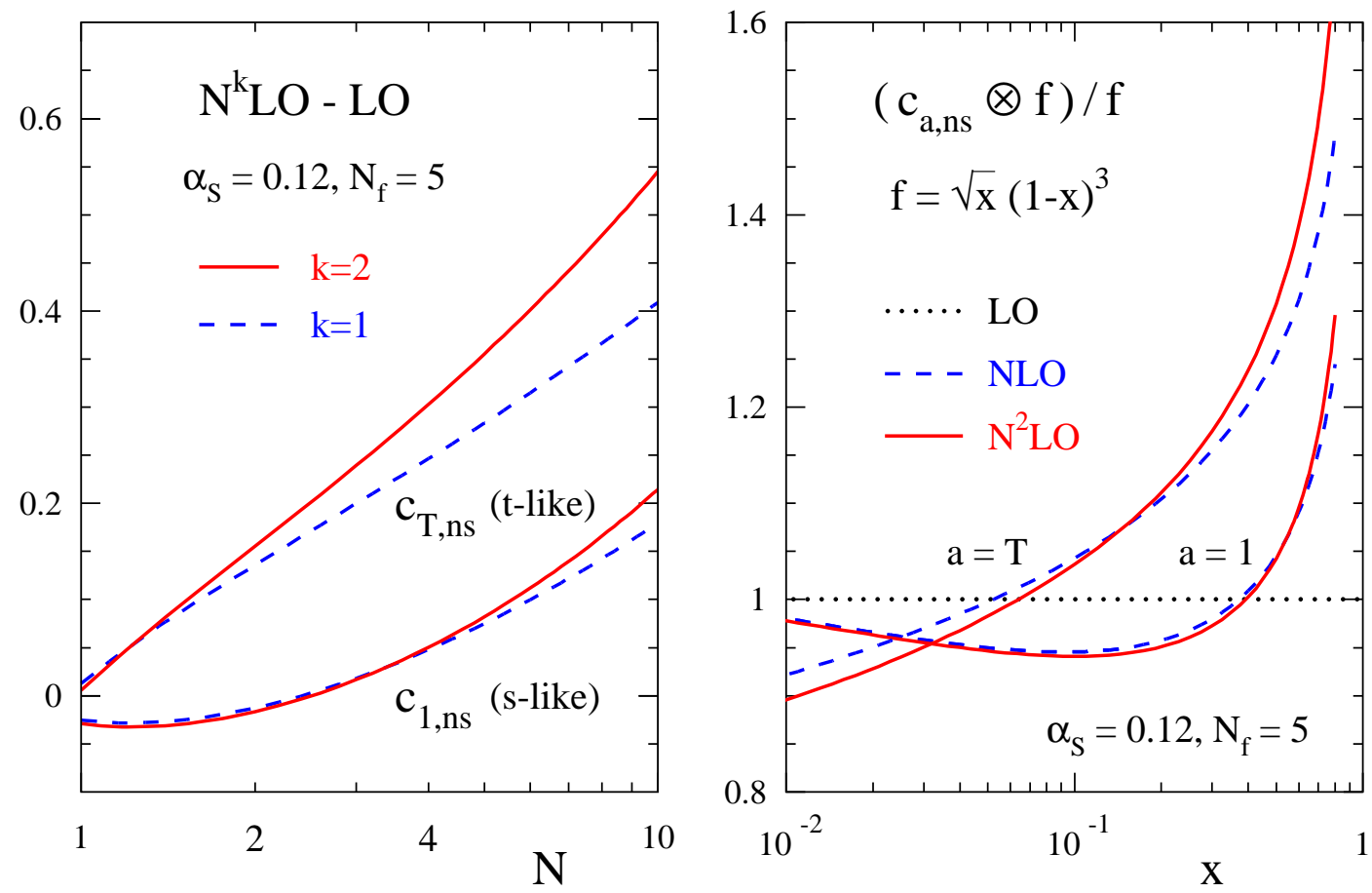

Figure 1: Comparison of the coefficient function $c_{T, \text { ns }}$ for the (time-like) process $e^{+} e^{-} \rightarrow h+X$ with its counterpart $c_{1, \text { ns }}$ in (space-like) deep-inelastic scattering for $Q^{2} \simeq M_{Z}^{2}$. Left plot: Mellin moments, right plot: convolutions (2) with a schematic input shape denoted by $f$.
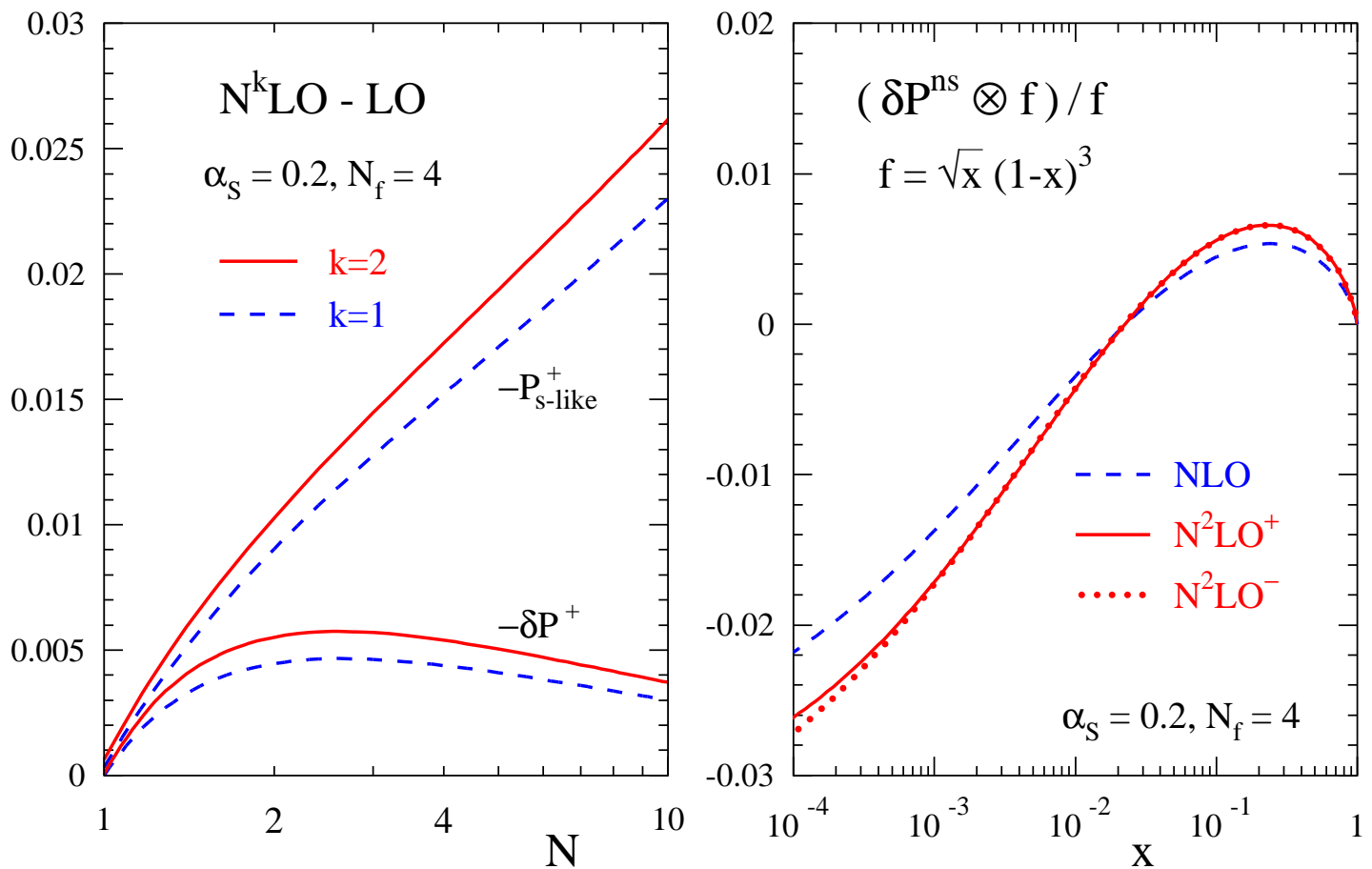

Figure 2: The differences $\delta P^{\mathrm{ns}}=P_{\sigma=1}^{\mathrm{ns}}-P_{\sigma=-1}^{\mathrm{ns}}$ between the time-like $(\sigma=1)$ and space-like $(\sigma=-1)$ non-singlet splitting functions at a 'low' scale characterized by the (order-independent) value $\alpha_{\mathrm{s}}=0.2$ of the strong coupling constant. Left: moment-space comparison with the higherorder corrections in the space-like case. Right: convolutions with a schematic input shape. 


\section{References}

[1] O. Biebel, P. Nason and B.R. Webber, hep-ph/0109282 and in S. Eidelman et al. [Particle Data Group], Phys. Lett. B592 (2004) 1

[2] P.J. Rijken and W.L. van Neerven, Phys. Lett. B386 (1996) 422, hep-ph/9604436

[3] P.J. Rijken and W.L. van Neerven, Nucl. Phys. B488 (1997) 233, hep-ph/9609377

[4] P.J. Rijken and W.L. van Neerven, Phys. Lett. B392 (1997) 207, hep-ph/9609379

[5] G. Curci, W. Furmanski and R. Petronzio, Nucl. Phys. B175 (1980) 27

[6] W. Furmanski and R. Petronzio, Phys. Lett. 97B (1980) 437

[7] E.G. Floratos, C. Kounnas and R. Lacaze, Nucl. Phys. B192 (1981) 417

[8] V.N. Gribov and L.N. Lipatov, Sov. J. Nucl. Phys. 15 (1972) 438, ibid. 675

[9] M. Stratmann and W. Vogelsang, Nucl. Phys. B496 (1997) 41, hep-ph/9612250

[10] D.J. Broadhurst and A.L. Kataev, Phys. Lett. B315 (1993) 179, hep-ph/9308274

[11] J. Blümlein, V. Ravindran and W.L. van Neerven, Nucl. Phys. B586 (2000) 349, hep-ph/0004172

[12] Y.L. Dokshitzer, G. Marchesini and G.P. Salam, Phys. Lett. B634 (2006) 504, hep-ph/0511302

[13] S. Moch, J.A.M. Vermaseren and A. Vogt, Nucl. Phys. B688 (2004) 101, hep-ph/0403192

[14] A. Vogt, S. Moch and J.A.M. Vermaseren, Nucl. Phys. B691 (2004) 129, hep-ph/0404111

[15] J.A.M. Vermaseren, A. Vogt and S. Moch, Nucl. Phys. B724 (2005) 3, hep-ph/0504242

[16] S. Moch, J.A.M. Vermaseren and A. Vogt, JHEP 0508 (2005) 049, hep-ph/0507039

[17] E. Remiddi and J.A.M. Vermaseren, Int. J. Mod. Phys. A15 (2000) 725, hep-ph/9905237

[18] S. Moch and J.A.M. Vermaseren, Nucl. Phys. B573 (2000) 853, hep-ph/9912355

[19] J.A.M. Vermaseren, math-ph/0010025

[20] J.A.M. Vermaseren, Int. J. Mod. Phys. A14 (1999) 2037, hep-ph/9806280

[21] S. Frixione and M. Grazzini, JHEP 0506 (2005) 010, hep-ph/0411399

[22] A. Mitov, hep-ph/0511340

[23] Y.L. Dokshitzer, V.A. Khoze and S.I. Troian, Phys. Rev. D53 (1996) 89, hep-ph/9506425

[24] J.A. Gracey, Phys. Lett. B322 (1994) 141, hep-ph/9401214

[25] S. Albino, B.A. Kniehl and G. Kramer, Nucl. Phys. B725 (2005) 181, hep-ph/0502188

[26] P. Aurenche, R. Basu, M. Fontannaz and R.M. Godbole, Eur. Phys. J. C34 (2004) 277, hep-ph/0312359

[27] A. Daleo, D. de Florian and R. Sassot, Phys. Rev. D71 (2005) 034013, 0411212

[28] B. Kniehl, G. Kramer and M. Maniatis, Nucl. Phys. B711 (2005) 345, E: ibid. B720 (2005) 231, hep-ph/0411300

[29] P. Aurenche, M. Fontannaz, J.P. Guillet, B. Kniehl and M. Werlen, Eur. Phys. J. C13 (2000) 347, hep-ph/9910252

[30] B. Kniehl, G. Kramer and B. Pötter, Nucl. Phys. B597 (2000) 337, hep-ph/0011155

[31] D. de Florian and W. Vogelsang, Phys. Rev. D71 (2005) 114004, hep-ph/0501258

[32] G. Corcella and A. D. Mitov, Nucl. Phys. B623 (2002) 247, hep-ph/0110319

[33] K. Melnikov and A. Mitov, Phys. Rev. D70 (2004) 034027, hep-ph/0404143

[34] A. Mitov, Phys. Rev. D71 (2005) 054021, hep-ph/0410205

[35] T. Gehrmann and E. Remiddi, Comput. Phys. Commun. 141 (2001) 296, hep-ph/0107173 